\title{
DIFFERENTIAL FORMS AND HOMOTOPY GROUPS
}

\author{
KUO-TSAI CHEN
}

The main result of this paper relates differential forms of a manifold to the fundamental group and its action on higher homotopy groups as follows:

Theorem. If a connected $C^{\infty}$ manifold $X$ possesses closed 1 -forms $w_{1}, \cdots$, $w_{m}$, such that

(a) $w_{i} \wedge w_{j}=0, i, j=1, \cdots, m$,

(b) $\left[w_{1}\right], \ldots,\left[w_{m}\right]$ are linearly independent,

then $\pi_{1}(X)$ contains a free subgroup $G$ of rank $m$. If, moreover, there exists a closed $q$-form $w, q>1$, satisfying the conditions:

(c) $w_{i} \wedge w=0,1=1, \cdots, m$,

(d) for some $\bar{\alpha} \in \pi_{q}(X)$, the integral $\int w$ is nonzero over $\bar{\alpha}$, then $\{\bar{\beta} \cdot \bar{\alpha}\}_{\beta \in G}$ is a basis of a free abelian subgroup $\pi_{q}(X)$.

Corollary 1. Let $X$ be a compact Kähler manifold, and let $b^{r, s}=$ $\operatorname{dim} H^{r, s}(X)$. If $b^{1,0}>b^{2,0}+1$, then $\pi_{1}(X)$ contains a free subgroup of rank 2 . If $b^{1,0}>b^{2,0}+b^{q+1,0}+1, q>1$, and there exists a closed holomorphic $q$-form $w$ with $\int_{\alpha} w \neq 0$ for some spherical $q$-chain $\alpha$, then $\pi_{q}(X)$ contains a free abelian subgroup of infinite rank.

Corollary 2. If a compact Kähler manifold $X$ has a nonconstant holomorphic map into a Riemann surface of genus $>1$, then $\pi_{1}(X)$ has a free subgroup of rank at least 2.

We use a method of iterated integrals [2], which now works not only for 1 -forms but also for forms of higher degrees. Useful integrals are obtained through an extended defining system for a Massey product of cocycles [4], [6].

We begin with an abstract formulation in $\S 1$ followed in $\S 2$ by a treatment of integrals which can be obtained through extended defining systems for Massey products. The language of categories is used so that the theory can be interpreted in terms of the singular cohomology theory, whose aspect is not treated in this paper. In $\S 3$, we consider iterated integrals of differential forms. The mentioned result will be divided into two theorems, which are given as applications in $\S 4$. For iterated integrals of forms of higher degrees only, our treatment does not go beyond mentioning the fact that J. H. C. Whitehead's integral for Hopf invariant in [11] can be taken as a twice iterated integral.

Received October 5, 1970. Supported in part by NSF GP-22929. 
For future reference our method is presented in $\S \S 1 \& 2$ in a rather general form. Those readers who are only interested in the main result of this paper may begin from $\S 3.4$.

\section{The abstract formulation}

The main purpose of this section is to set up a frame work in which iterated path integration is possible. We first choose a family of parameter spaces each of which has a cochain complex which can be extended to an acyclic one. Integration along a path (i.e., a map from a parameter space to a manifold) can be carried out in the parameter space whose acyclic condition makes way for iterated integration.

1.1. Let $k$ be a commutative ring, and $\sqrt{ }$ a category which is equipped with a contravariant functor $C^{*}$ from $(5$ to the category of differential graded $k$ algebras (or simply, DG $k$-algebras). The differential of $C^{*}$ will be denoted by $d$ and the product by $U$. The associated cocycle, coboundary and cohomology functors on the category 5 will be respectively denoted by $Z^{*}, B^{*}$ and $H^{*}$.

Assume that the category $\mathfrak{C}$ is further equipped with a family $\mathfrak{P}$ of objects called parameter objects. For each parameter object $Y$, we shall assume that the DG $k$-algebra $C^{*}(Y)$ can be extended to a DG $k$-algebra $C_{\mathfrak{B}}^{*}(Y)$ satisfying the following conditions:

(a) $C_{\mathfrak{\beta}}^{*}(Y)$ is acyclic, i.e., all cohomology groups vanish.

(b) $C^{*}(Y)$ is an ideal of the DG $k$-algebra $C_{\mathfrak{\beta}}^{*}(Y)$.

Regard $\mathfrak{P}$ as a full subcategory of $\mathfrak{F}$, and take $C^{*}$ and $C_{\mathfrak{P}}^{*}$ both as functors from $\mathfrak{P}$ to the category of DG $k$-algebras. We further demand that

(c) the inclusion $C^{*} \subset C_{\Re}^{*}$ is natural.

1.2. Let $X$ be a given object of $\mathfrak{E}$, and $Y, Y^{\prime}$ arbitrary parameter objects. Let $\alpha: Y \rightarrow X$ be a morphism. The set of such morphisms will be denoted by Mor $(Y, X)$. Regard Mor $(\ldots, X)$ and $H^{q}(\ldots)$ as contravariant functors from the category $\mathfrak{B}$ to the category of sets.

Denote by $\bar{H}^{q}(X)$ the totality of natural transformations from Mor $(\ldots, X)$ to $H^{q}(\ldots)$. In other words, if $w \in \tilde{H}^{q}(X)$, then, for each $\alpha$, there is an assigned element $w_{\alpha}$ of $H^{q}(Y)$, and for each commutative triangle in $\mathbb{5}$

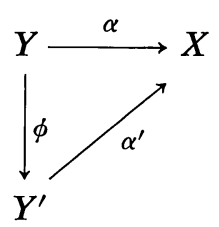

we have $H^{*}(\phi) w_{\alpha^{\prime}}=w_{\alpha}$. There is an obvious $k$-module structure on $\bar{H}^{q}(X)$.

Alternately $\bar{H}^{q}(X)$ can be constructed from cochains as follows:

Let $\bar{C}^{q}(X)$ denote the set of all maps 


$$
w: \bigcup_{Y \in \mathfrak{R}} \operatorname{Mor}(Y, X) \rightarrow \bigcup_{Y \in \mathfrak{B}} C^{q}(Y)
$$

such that $w$ sends every $\alpha: Y \rightarrow X$ to $w_{\alpha} \in C^{q}(X)$. Then $\bar{C}^{q}(X)$ has an obvious $k$-module structure. Define $d: \bar{C}^{q}(X) \rightarrow \bar{C}^{q+1}(X)$ such that $(d w)_{\alpha}=d w_{\alpha}$. Let $\tilde{Z}^{q}(X)$ be the submodule of $\tilde{C}^{q}(X)$ consisting of all $w$ with $d w=0$ such that, for any commutative triangle (1.2.1),

$$
C^{*}(\phi) w_{\alpha^{\prime}}=w_{\alpha} \quad \bmod d C^{q-1}(Y) .
$$

Then $\bar{H}^{q}(X) \approx \tilde{Z}^{q}(X) / d \hat{C}^{q-1}(X)$, which will be hereafter taken as the definition of $\hat{H}^{q}(X)$.

1.3. An element $w \in \bar{C}^{q}(X)$ is said to be coherent if

$$
C^{*}(\phi) w_{\alpha^{\prime}}=w_{\alpha}
$$

for every commutative triangle (1.2.1).

There is natural homomorphism

$$
C^{q}(X) \rightarrow \bar{C}^{q}(X)
$$

which sends each element $w$ of $C^{q}(X)$ to a coherent element also denoted by $w$ such that $w_{\alpha}=C^{*}(\alpha) w$. This homomorphism induces a natural homomorphism

$$
H^{q}(X) \rightarrow \bar{H}^{q}(X)
$$

1.4. Similar to $\bar{C}^{q}(X)$, we define $\bar{C}_{\mathfrak{P}}^{q}(X)$ so that, if $w \in \bar{C}_{\mathfrak{P}}^{q}(X)$, then $w_{\alpha}$ belongs to $C_{\mathfrak{P}}^{q}(Y)$ instead of $C^{q}(Y)$. Then $\tilde{C}^{q}(X)$ is a $k$-submodule of $\tilde{C}_{\mathfrak{\beta}}^{q}(X)$.

If $u \in \tilde{C}^{q}(X), q>0$, such that $d u=0$, then $u \in d \check{C}_{\Re}^{q-1}(X)$. An arbitrary element $v \in \bar{C}_{\mathfrak{P}}^{q-1}(X)$ with $d v=u$ will be written $v=\int u$, which is unique up to a difference in $d \overleftarrow{C}_{\Re}^{q-2}(X)$ and therefore unique when $q=1$.

Observe that, since $C^{*}(Y)$ is an ideal of $C_{\mathfrak{\beta}}^{*}(Y),\left(\int u\right) \cup w$ is an element of $\bar{C}^{q+r-1}(X)$ for any $w \in \bar{C}^{r}(X)$. Observe also that, if $d w=0$, then

$$
\left.d\left(\int u\right) \cup w\right)=u \cup w
$$

If $U$ is a submodule of $\bar{C}^{q}(X)$ such that $d U=0$, then $\int U$ will denote the submodule of $\bar{C}_{\mathfrak{\beta}}^{q-1}(X)$ of all $\int u, u \in U$.

1.5. We are going to give an abstract version of interated integration. Let there be given submodules $W^{q} \subset \bar{C}^{q}(X), q>0$, each of which consists of coherent elements. What we have particularly in mind as an example is the 
case of $W^{q}$ being the image of the natural homomorphism $C^{q}(X) \rightarrow \bar{C}^{q}(X)$.

Construct an ascending filtration $F$ of $\bar{C}^{q}(X)$ and $\tilde{Z}^{q}(X), q \geq 1$, such that

$$
\begin{aligned}
F^{1} \tilde{C}^{q}(X)=W^{q}, \quad F^{1} \tilde{Z}^{q}(X) & =W^{q} \cap \tilde{Z}^{q}(X), \text { and, for } r \geq 2, \\
F^{r} \tilde{C}^{q}(X) & =\sum_{i=1}^{q}\left(\int F^{r-1} \tilde{Z}^{q-i+1}(X)\right) \cup W^{i}+F^{r-1} \tilde{C}^{q}(X), \\
F^{r} \tilde{Z}^{q}(X) & =F^{r} \bar{C}^{q}(X) \cap \tilde{Z}^{q}(X) .
\end{aligned}
$$

Set

$$
F^{r} \hat{H}^{q}(X)=\left(F^{r} \tilde{Z}^{q}(X)+d \hat{C}^{q-1}(X)\right) / d \bar{C}^{q-1}(X)
$$

1.6. For the purpose of illustration, we consider a simple example. Let $\sqrt{5}$ be the category of pointed connected $C^{\infty}$ manifolds, the real line $R$ with 0 as its base point be the single parameter object, $X$ be a $C^{\infty}$ manifold with $x_{0}$ as its base point, $k$ be the real number field, and $C^{*}(X)$ be the exterior algebra on $X$ such that $C^{0}(X)$ consists of all $C^{\infty}$ functions having a zero at $x_{0}$. Set $C_{\mathfrak{\beta}}^{*}(R)$ $=C^{*}(R)$.

Since $Z^{1}(R)=C^{1}(R)$ and $C^{q}(R)=0, q>1$, we have $\tilde{Z}^{1}(X)=\check{C}^{1}(X)$ and $\bar{C}^{q}(X)=0, q>1$.

The natural homomorphism $C^{1}(X) \rightarrow \bar{C}^{1}(X)$ is injective, and its image is taken to be $W^{1}$. Let the graded module $W$ be such that $W^{q}=0$ for $q \neq 1$. The elements of $F^{1} \bar{C}^{1}(X)=F^{1} \tilde{Z}^{1}(X)=W^{1}$, may be identified with 1-forms on $X$.

Let $w, w_{1}, \cdots \in W^{1}$ be 1 -forms. For a base point preserving $C^{\infty} \operatorname{map} \alpha: R \rightarrow X$, write

$$
\alpha^{*}=C^{1}(\alpha): C^{1}(X) \rightarrow C^{1}(R)
$$

If $v \in C^{1}(R)$, let $\int_{0} v$ be the unique $C^{\infty}$ function $f$ on $R$ such that $d f=v$ and $f(0)=0$. Define the iterated integrals $\int_{x_{0}} w_{1} \cdots w_{r} \in \tilde{C}^{0}(X)$ such that $\left(\int_{x_{0}} w_{1}\right)_{\alpha}=$ $\int_{0} \alpha^{*} w_{1}$, and for $r \geq 2$,

$$
\left(\int_{x_{0}} w_{1} \cdots w_{r}\right)_{\alpha}=\int_{0}\left(\int_{x_{0}} w_{1} \cdots w_{r-1}\right)_{\alpha} \alpha^{*} w_{r}
$$

Set $\int_{x_{0}} w_{1} \cdots w_{r}=1$, when $r=0$. Then each $\int_{x_{0}} w_{1} \cdots w_{r}$ is a coherent element of $\underset{\tilde{C}_{\mathfrak{\beta}}^{0}}{\stackrel{x_{0}}{(X)}}(X)$, and

$$
d \int_{x_{0}} w_{1} \cdots w_{r}=\left(\int_{x_{0}} w_{1} \cdots w_{r-1}\right) \cup w_{r} \in F^{r} \bar{C}^{1}(X)=F^{r} \tilde{Z}^{1}(X),
$$


for $r \geq 1$. As a matter of fact, $F^{r} \bar{C}^{1}(X)$ is spanned by elements of the types $d \int_{x_{0}} w_{1}, d \int_{x_{0}} w_{1} w_{2}, \cdots, d \int_{x_{0}} w_{1} \cdots w_{r}$.

\section{Iterated integrals}

2.1. Let $w_{i}$ be a coherent element of $\tilde{Z}^{q_{i}}(X), q_{i} \geq 1, i=1, \cdots, r$. Set

$$
q_{i j}=q_{i}+\cdots+q_{j}-j+i
$$

so that $q_{i i}=q_{i}$. Set $\varepsilon(i, j)=(-1)^{q_{i j}}$, and note that $\varepsilon(i, j) \varepsilon(j+1, k)=-\varepsilon(i, k)$ for $i<j<k$.

A Massey system (or a defining system for a Massey product) of $w_{1}, \cdots, w_{r}$ is a set of coherent elements $w(i, j) \in \bar{C}^{q_{i j}}(X), 1 \leq i<j \leq r,(i, j) \neq(1, r)$, satisfying the conditions:

$$
d w(i, j)=\sum_{i \leq l \leq j} \varepsilon(i, l) w(i, l) \cup w(l+1, j),
$$

where $w(i, i)=w_{i}$.

The element

$$
\begin{aligned}
z= & \varepsilon(1,1) w(1,1) \cup w(2, r)+\varepsilon(1,2) w(1,2) \cup w(3, r) \\
& +\cdots+\varepsilon(1, r-1) w(1, r-1) \cup w(r, r)
\end{aligned}
$$

is a coherent element of $\tilde{Z}^{q_{1 r+1}}(X)$ and is called a Massey product of type $\left\langle w_{1}, \cdots, w_{r}\right\rangle$.

An extended Massey system of $w_{1}, \cdots, w_{r}$ is a Massey system together with a coherent element $w(1, r) \in \check{C}^{q_{1} r}(X)$ such that (2.1.1) holds for $1 \leq i<j \leq r$. In this case, $z=d w(1, r) \in d \bar{C}^{q_{1 r}}(X)$.

For most applications mentioned in this work, we shall only use extended Massey systems of the trivial type such that $w(i, j)=0,1 \leq i<j \leq r$. The only condition required for $w_{1}, \cdots, w_{r}$ is that $w_{i} \cup w_{i+1}=0,1 \leq i<r$.

2.2. For an extended Massey system of $w_{1}, \cdots, w_{r}$, define inductively

$$
u(1,1)=-\varepsilon(1,1) w_{1}, \quad v(1,1)=\int u(1,1)=-\varepsilon(1,1) \int w_{1}
$$

and, for $1<j \leq r$,

$$
u(1, j)=-\varepsilon(1, j)\left[\sum_{1 \leq i<j} v(1, i) \cup w(i+1, j)+w(1, j)\right] .
$$

It will be shown shortly that $d u(1, j)=0$ so that we may define $v(1, j)=$ $\int u(1, j) \in \bar{C}_{\mathfrak{\beta}}^{q_{1 j^{-1}}}(X)$. 
If the extended Massey system is of the trivial type, and if, for $j>1$, we define $\int w_{1} \cdots w_{j}=\int\left(\left(\int w_{1} \cdots w_{j-1}\right) \cup w_{j}\right)$, then

$$
v(1, j)=(-1)^{j} \varepsilon(1,1) \varepsilon(1,2) \cdots \varepsilon(1, j) \int w_{1} \cdots w_{j}
$$

Let $W^{q}, q>0$, be given submodules of coherent elements of $\bar{C}^{q}(X)$, which give rise to the filtrations $\left\{F^{r} \bar{C}^{q}(X)\right\}$ and $\left\{F^{r} \tilde{Z}^{q}(X)\right\}$ as described in $\S 1.5$. By an extended Massey system of $w_{1}, \cdots, w_{r}$ in $W$, we mean a Massey system which satisfies the additional condition that $w(i, j) \in W^{q_{i j}}, 1 \leq i \leq j \leq r$.

Theorem. For any extended Massey system of $w_{1}, \cdots, w_{r}$ in $W$, elements $u(1, r)$ exist and belong to $F^{r} \tilde{Z}^{q_{1 r}}(X)$,

We shall call $v(1, r)$ an iterated integral of the type $\left\langle w_{1}, \cdots w_{r}\right\rangle$.

Corollary. For any extended Massey system of the trivial type of $w_{1}, \cdots$, $w^{r}$ in $W, \int w_{1} \cdots w_{r}$ exists and belongs to $F^{r} \tilde{Z}^{q_{1}}(X)$.

The proof of the theorem is computational and is divided into two parts, which are respectively presented in $\S 2.3$ and $\S 2.4$. The computation can be simplified if we restrict ourselves only to extended Massey systems of the trivial type, which is sufficient for our main results.

2.3. We are going to show by indution on $r$ that $u(1, r)$ is a well defined element of $\bar{C}^{q_{1} r}(X)$ and that $d u(1, r)=0$. For $r>1$, it follows from the induction hypotheses that $v(1, r-1) \in \bar{C}_{\mathfrak{B}}^{q_{1 r-1^{-1}}}(X)$ and that $u(1, r) \in \bar{C}^{q_{1 r}}(X)$. Moreover,

$$
\begin{aligned}
-\varepsilon(1, r) d u(1, r)= & d\left(\sum_{1 \leq i<r} v(1, i) \cup w(i+1, r)\right)+d w(1, r) \\
= & \sum_{1 \leq i<r} u(1, i) \cup w(i+1, r) \\
& -\sum_{1<i<r} \varepsilon(1, i) v(1, i) \cup d w(i+1, r)+d w(1, r), \quad \text { by }(2.1 .1) \\
= & \sum_{1 \leq j<r} u(1, j) \cup w(j+1, r) \\
& +\sum_{1 \leq i<j<r} \varepsilon(1, j) v(1, i) \cup w(i+1, j) \cup w(j+1, r) \\
& +\sum_{1<j<r} \varepsilon(1, j) w(1, j) \cup w(j+1, r), \quad \text { by }(2.2 .1) \\
= & 0 .
\end{aligned}
$$

2.4. We need to show that $u(1, r) \in \tilde{Z}^{q_{1 r}}(X)$. Then the fact that $u(1, r) \in F^{r} \tilde{Z}^{q_{1 r}}(X)$ follows from the definition of $u(1, r)$.

Let a commutative triangle (1.2.1) be given, and set 


$$
\begin{aligned}
& \Delta u(1, i)=C^{*}(\phi) u(1, i)_{\alpha^{\prime}}-u(1, i)_{\alpha} \in C^{q_{1 r}}(Y), \\
& \Delta v(1, i)=C_{\Re}^{*}(\phi) v(1, i)_{\alpha^{\prime}}-v(1, i)_{\alpha} \in C_{\Re}^{q_{1 i}-1}(Y) .
\end{aligned}
$$

We are going to prove by induction on $r \geq 2$ that there exists $c(1, i) \in C_{\Re}^{q_{1 i}-^{2}}(Y)$, $1 \leq i \leq r$, such that

$$
\begin{aligned}
& \Delta u(1, r)=-\varepsilon(1, r) d\left(\sum_{1 \leq i<r} c(1, i) \cup w(i+1, r)_{\alpha}\right), \\
& \Delta v(1, r)=-\varepsilon(1, r) \sum_{1 \leq i<r} c(1, i) \cup w(i+1, r)_{\alpha}+d c(1, r)_{\alpha^{\prime}} .
\end{aligned}
$$

Take note that (2.4.1) implies $u(1, r) \in \tilde{Z}^{q_{1 r}}(X)$.

Recall that if $u(1, i) \in \tilde{Z}^{\prime}(X)$, then $v(1, i)=\int_{x_{0}} u(1, i)$ is unique.

For $r=1$, we have $d \Delta v(1,1)=\Delta u(1,1)=0$. Choose any $c(1,1) \in C_{\mathfrak{P}}^{q_{11}-2}(Y)$ such that $\Delta v(1,1)=d c(1,1)$. For $r>1$, according to (2.2.1),

$$
\begin{gathered}
-\varepsilon(1, r) \Delta u(1, r)=\sum_{1 \leq j<r} \Delta v(1, j) \cup w(j+1, r)_{\alpha} \\
\quad \text { by the induction hypothesis } \\
=\sum_{1 \leq i<j<r}-\varepsilon(1, j) c(1, i) \cup w(i+1, j)_{\alpha} \cup w(j+1, r)_{\alpha} \\
\quad+\sum_{1 \leq j<r} d c(1, j) \cup w(j+1, r)_{\alpha} \\
=\sum_{1 \leq i<r} \varepsilon(1, i) c(1, i) \cup d w(i+1, r)_{\alpha}+\sum_{1 \leq i<r} d c(1, i) \cup w(i+1, r)_{\alpha} \\
=d \sum_{1 \leq i<r} c(1, i) \cup w(i+1, r)_{\alpha} .
\end{gathered}
$$

Consequently, there exists $c(1, r) \in C_{\mathfrak{\beta}}^{q_{1 r^{-2}}}(Y)$ such that

$$
\Delta v(1, r)=-\varepsilon(1, r) \sum_{1 \leq i<r} c(1, i) \cup w(i+1, r)_{\alpha}+d c(1, r) .
$$

Hence the theorem is proved.

\section{Integrals of differential forms}

3.1. Let $I$ be the unit interval, and the $n$-cube $I^{n}$ be given the $C^{\infty}$ structure of that of a subset of $R^{n}$. By a $C^{\infty}$ manifold pair, we mean a pair $(X, A)$, where $X$ is a connected $C^{\infty}$ manifold and $A$ a closed submanifold.

Let $k$ be the real (or complex) number field, and $C^{*}(X)$ be the real (or complex) exterior algebra on $X$ whose exterior multiplication will be denoted by $\wedge$. The exterior algebra $C^{*}(X, A)$ is defined to be the kernel of the homomorphism $C^{*}(X) \rightarrow C^{*}(A)$ induced by the inclusion $A \subset X$. 
Let $\subseteq$ be the category whose objects are $C^{\infty}$ manifold pairs together with $\left(I^{n}, \partial I^{n}\right)$ and $\left(I^{n} \times I, \partial I^{n} \times I\right), n \geq 1$, and whose morphisms are $C^{\infty}$ maps of pairs. Let $0=(0, \cdots, 0) \in I^{n}$.

Let $\mathfrak{P}$ be the family of $\left(I^{n}, \partial I^{n}\right)$ and $\left(I^{n} \times I, \partial I^{n} \times I\right), n \geq 1$. Set $C_{\mathfrak{P}}^{*}\left(I^{n}, \partial I^{n}\right)$ $=C^{*}\left(I^{n}, 0\right)$ and $C_{\mathfrak{\beta}}^{*}\left(I^{n} \times I, \partial I^{n} \times I\right)=C^{*}\left(I^{n} \times I, 0\right)$. This choice of parameter category $\mathfrak{P}$ is designed to relate $\hat{H}^{*}$ with homotopy groups $\pi_{n}$.

3.2. Let $(X, A)$ be a $C^{\infty}$ manifold pair, and $q$ a positive integer. If $w \in C^{q}(X, A)$ is a $q$-form, its image under the canonical homomorphism $C^{q}(X, A) \rightarrow \bar{C}^{q}(X, A)$ will also be denoted by $w$. (As a matter of fact, this map is injective.) We shall keep referring to $w \in \bar{C}^{q}(X, A)$ as a $q$-form.

Let $\alpha:\left(I^{q}, \partial I^{q}\right) \rightarrow(X, A)$ denote an arbitrary $C^{\infty}$ map. If $w \in \bar{C}^{q}(X, A)$, set

$$
\int_{\alpha} w=\int_{I^{q}} w_{\alpha}
$$

Proposition. Let $w \in \tilde{Z}^{q}(X, A)$. If two $C^{\infty}$ maps $\alpha_{i}:\left(I^{q}, \partial I^{q}\right) \rightarrow(X, A)$, $i=0,1$, are homotopic, then

$$
\int_{\alpha_{0}} w=\int_{\alpha_{1}} w
$$

Proof. Let $h:\left(I^{q} \times I, \partial I^{q} \times I\right) \rightarrow(X, A)$ be a $C^{\infty}$ homotopy from $\alpha_{0}$ to $\alpha_{1}$. Then $\int_{\partial\left(I q^{q} I\right)} w_{h}=\int_{\partial I^{q} \times I} w_{h}=0$, so that

$$
\int_{I^{q} \times\{0\}} w_{h}=\int_{I^{q} \times\{1\}} w_{h} .
$$

Let $\phi$ denote the composite map $I^{q} \rightarrow I^{q} \times\{0\} \subset I^{q} \times I$, so that $h \phi=\alpha_{0}$. Since $w \in \tilde{Z}^{q}(X, A)$,

$$
C^{*}(\phi) w_{h}=w_{\alpha_{0}} \quad \bmod B^{q}\left(I^{q}, \partial I^{q}\right) .
$$

Hence $\int_{\alpha_{0}} w=\int_{I^{q \times\{0\}}} w_{h}$, and similarly, $\int_{\alpha_{1}} w=\int_{I^{q \times\{1\}}} w_{h}$.

The homotopy class of $\alpha:\left(I^{q}, \partial I^{q}\right) \rightarrow(X, A)$ will be denoted by $\bar{\alpha}$. We are justified to define

$$
\int_{\alpha} w=\int_{\alpha} w
$$

for $w \in \tilde{Z}^{q}(X, A)$.

3.3. Let $w_{1}, \cdots, w_{r} \in \tilde{Z}^{1}\left(X, x_{0}\right)$ such that $w_{1} \wedge w_{2}=\cdots=w_{r-1} \wedge w_{r}=0$. 
According to $\S 2.1$, this gives rise to an extended Massey system of the trivial type. We have $\int w_{1} \cdots w_{r} \in \check{C}_{\mathfrak{P}}^{0}\left(X, x_{0}\right)$. Since $\int w_{1} \cdots w_{r}=\int_{x_{0}} w_{1} \cdots w_{r}$ is unique, it is a coherent element of $\bar{C}_{\Re}^{0}\left(X, x_{0}\right)$. For $\alpha:(I, \partial I) \rightarrow \stackrel{x_{0}}{\rightarrow}\left(X, x_{0}\right)$, define $\int_{\alpha} w_{1} \cdots w_{r}$ to be the value of the function $\left(\int w_{1}, \cdots, w_{r}\right)_{\alpha}$ at the point $1 \in I$. In other words, for $r \geq 2$,

$$
\int_{\alpha} w_{1} \cdots w_{r}=\int_{\alpha}\left(\int w_{1} \cdots w_{r-1}\right) \wedge w_{r} .
$$

Then the value of $\int_{\alpha} w_{1} \cdots w_{r}$ depends only on the homotopy class $\bar{\alpha}$.

3.4. Let $\alpha:[0,1] \rightarrow X$ be a path, and $w_{1}, \cdots, w_{r}$ be 1 -forms. Write $\alpha^{*} w_{i}$ $=f_{i}(t) d t$. Then iterated path integrals can be explicitly given by

$$
\int_{\alpha} w_{1} \cdots w_{r}=\int_{0}^{1} \int_{0}^{t_{r}} \cdots \int_{0}^{t_{2}} f_{1}\left(t_{1}\right) d t_{1} \cdots f_{r-1}\left(t_{r-1}\right) d t_{r-1} f_{r}\left(t_{r}\right) d t_{r} .
$$

Recall that if $w_{1}, \cdots, w_{r}$ are closed 1-forms such that $w_{1} \wedge w_{2}=\cdots=$ $w_{r-1} \wedge w_{r}=0$, then the value of $\int_{\alpha} w_{1} \cdots w_{r}$ depends only on the homotopy class of the path $\alpha$ (see also [2]).

In particular, if $\alpha$ is a product $\beta \gamma$, then

$$
\begin{aligned}
\int_{\alpha} w_{1} \cdots w_{r}= & \int_{r} w_{1} \cdots w_{r}+\cdots+\int_{\beta} w_{1} \cdots w_{i} \int_{r} w_{i+1} \cdots w_{r} \\
& +\cdots+\int_{\beta} w_{1} \cdots w_{r} .
\end{aligned}
$$

Recall also the formula

$$
\int_{\alpha} w_{1} \cdots w_{r} \int_{\alpha} w_{r+1} \cdots w_{r+s}=\sum_{\sigma} \int_{\alpha} w_{\sigma 1} \cdots w_{\sigma(r+s)}
$$

summing over all $(r, s)$-shuffles $\sigma$, i.e., permutations of $r+s$ letters satisfying

$$
\sigma^{-1} 1<\cdots<\sigma^{-1} r, \quad \sigma^{-1}(r+1)<\cdots<\sigma^{-1}(r+s) .
$$

A more detailed account of formulas (3.4.1) and (3.4.2) can be found in $[2, \S 1.5-\S 1.6]$. 
3.5. We are going to establish a useful proposition concerning formal power series.

Let $k[[X]]$ denote the $k$-algebra of formal power series of noncommutative indeterminates $X_{1}, \cdots, X_{m}$, and $A$ be the $k$-algebra of formal power series of indeterminates $X_{1}^{\prime}, \cdots, X_{m}^{\prime}, X_{1}^{\prime \prime}, \ldots, X_{m}^{\prime \prime}$ which are noncommutative except the relations $X_{i}^{\prime} X_{i}^{\prime \prime}=X_{j}^{\prime \prime} X_{i}^{\prime}, i, j=1, \cdots, m$. Let

$$
\Delta: k[[X]] \rightarrow A
$$

be the obvious $k$-algebra homomorphism given by

$$
X_{i} \mapsto X_{i}^{\prime}+X_{i}^{\prime \prime}, \quad i=1, \cdots, m,
$$

and $\rho^{\prime}$ and $\rho^{\prime \prime}: k[[x]] \rightarrow A$ be the obvious $k$-algebra homomorphism given respectively by $X_{i} \mapsto X_{i}^{\prime}$ and $X_{i} \mapsto X_{i}^{\prime \prime}$. For $u \in k[[X]]$, write $u^{\prime}=\rho^{\prime} u$ and $u^{\prime \prime}=\rho^{\prime \prime} u$.

Definition. An element $g \in k[[X]]$ is grouplike if $\Delta g=g^{\prime} g^{\prime \prime}$.

Lemma. If $g_{1}, \cdots, g_{r}$ are linearly independent grouplike elements, then $g_{i}^{\prime} g_{j}^{\prime \prime}, i, j=1, \cdots, r$, are linearly independent over $k$.

Proof. Let $\rho_{i_{1} \cdots i_{l}}: k\left[\left[X^{\prime \prime}\right]\right] \rightarrow k$ send every element of $k\left[\left[X^{\prime \prime}\right]\right]$ to its coefficient of the term $X_{i_{1}}^{\prime \prime} \ldots X_{i l}^{\prime \prime}$, and $c_{i j} \in k$ be such that $\sum_{i, j} c_{i j} g_{i}^{\prime} g_{j}^{\prime \prime}=0$. Then $\sum_{i}\left(\sum_{j} c_{i j}\left(\rho_{i_{1} \cdots i_{l}} g_{j}^{\prime \prime}\right)\right) g_{i}^{\prime}=0$ which implies that, for every $i, i_{1}, \cdots, i_{l}$,

$$
\rho_{i_{1} \cdots i_{l}}\left(\sum_{j} c_{i j} g_{j}^{\prime \prime}\right)=0
$$

Hence $\sum_{j} c_{i j} g_{j}^{\prime \prime}=0$, i.e., $c_{i j}=0$.

The above lemma enables us to prove the next assertion in the same way as for the case of grouplike elements of Hopf algebras [10].

Proposition. If $g_{0}, \cdots, g_{r}$ are distinct grouplike elements of $k[[X]]$, then they are linearly independent over $k$.

Proof. Use induction on $r \geq 0$. For $r>0$, suppose that

$$
g_{0}=c_{1} g_{1}+\cdots+c_{r} g_{r}, \quad c_{i} \in k
$$

Since by the induction hypothesis, $g_{1}, \ldots, g_{r}$ are linearly independent, so are $g_{i}^{\prime} g_{j}^{\prime \prime}, i, j=1, \cdots, r$. We have

$$
\left(c_{1} g_{1}^{\prime}+\cdots+c_{r} g_{r}^{\prime}\right)\left(c_{1} g_{1}^{\prime \prime}+\cdots+c_{r} g_{r}^{\prime \prime}\right)=c_{1} g_{1}^{\prime} g_{1}^{\prime \prime}+\cdots+c_{r} g_{r}^{\prime} g_{r}^{\prime \prime},
$$

which implies $c_{i} c_{j}=0, i \neq j$, and $c_{i}^{2}=c_{i}$ which forces the contradiction $g_{0}=g_{i}$ for some $i, 1 \leq i \leq r$. 


\section{Applications}

4.1 Theorem. If a $C^{\infty}$ manifold $X$ has closed 1 -forms $w_{1}, \cdots, w_{m}$ such that

(a) $w_{i} \wedge w_{j}=0, i, j=1, \cdots, m$,

(b) the cohomology class $\left[w_{1}\right], \cdots,\left[w_{m}\right]$ are linearly independent, then $\pi_{1}(X)$ contains a free subgroup of rank $m$.

Proof. There exist smooth loops $\beta_{1}, \cdots, \beta_{n}$ at $x_{0}$ such that the $m \times m$ matrix $\left(\int_{\beta j} w_{i}\right)$ is nonsingular. We may assume that $\int_{\beta j} w_{i}=\delta_{i j}$.

For a loop $\beta$ at $x_{0}$, let $\theta(\bar{\beta})$ be the formal power series

$$
1+\sum_{i}\left(\int_{\beta} w_{i}\right) X_{i}+\sum_{i, j}\left(\int_{\beta} w_{i} w_{j}\right) X_{i} X_{j}+\cdots .
$$

It follows from (3.4.1) that if $\beta$ is a product $\beta^{\prime} \beta^{\prime \prime}$, then

$$
\theta(\bar{\beta})=\theta\left(\bar{\beta}^{\prime}\right) \theta\left(\bar{\beta}^{\prime \prime}\right)
$$

Thus there is a multiplicative homomorphism

$$
\theta: \pi_{1}(X) \rightarrow k[[X]]
$$

given by $\bar{\beta} \mapsto \theta(\bar{\beta})$.

Since $\theta\left(\bar{\beta}_{i}\right)=1+X_{i}+$ higher degree terms, there exists an automorphism $\chi$ of the $k$-algebra $k[[X]]$ such that

$$
\chi\left(\theta\left(\bar{\beta}_{i}\right)-1\right)=X_{i}, \quad i=1, \cdots, m .
$$

(See, for example, [1].) Thus

$$
\theta^{\prime}=\chi \theta: \pi_{1}(X) \rightarrow k[[X]]
$$

is the homomorphism given by

$$
\theta^{\prime}\left(\bar{\beta}_{i}\right)=1+X_{i}
$$

It is known [5] that, with respect to the multiplication, $1+X_{1}, \cdots, 1+X_{m}$ form a basis for a free group. In fact, the formal power series expansion of every reduced word $\left(1+X_{i_{1}}\right)^{n_{1}} \ldots\left(1+X_{i_{r}}\right)^{n_{r}}$ has a nonzero coefficient for the term $X_{i_{1}} \cdots X_{i_{r}}$. Hence the elements $\bar{\beta}_{i}, 1 \leq i \leq m$, generate a free subgroup.

Corollary. If $X$ is a compact Kähler manifold and $b^{1,0}>b^{2,0}+1$, then $\pi_{1}(X)$ contains a free-subgroup of rank at least 2.

Proof. Let $l=b^{1,0}$. Let $w_{1}, \cdots, w_{l}$ be linearly independent holomorphic closed 1-forms on $X$. Since $l-1>b^{2,0}$, the holomorphic 2-forms $w_{1} \wedge w_{2}$, 
$\cdots, w_{1} \wedge w_{l}$ must be linearly dependent. Hence we may assume that $w_{1} \wedge w_{2}$ $=0$ and apply the theorem.

Remark. The inequality in the above corollary can be written as $b_{1}<b_{2}+1$, where $b_{1}$ and $b_{2}$ are Betti numbers of $X$. An algebraic result of Stallings [8] implies that, for $K(\pi, 1)$, the condition $b_{2}=0$ is sufficient for $\pi$ to have a free subgroup of rank equal to $b_{1}$. The precise relation of his result with ours is not clear.

Corollary. If a compact Kähler manifold $X$ has a nonconstant holomorphic map into a Riemann surface of genus $>1$, then $\pi_{1}(X)$ has a free subgroup of rank at least 2.

Proof. There exist two linearly independent holomorphic 1-forms $w_{1}$ and $w_{2}$ on the Riemann surface $Y$ such that $w_{1} \wedge w_{2}=0$. If $f: X \rightarrow Y$ is a nonconstant holomorphic map, then $f^{*} w_{1}$ and $f^{*} w_{2}$ are linearly independent closed holomorphic 1-forms on $X$ with $f^{*} w_{1} \wedge f^{*} w_{2}=0$. According to the theorem, $\pi_{1}(X)$ has a free subgroup of rank 2 .

4.2. Let $w_{1}, \cdots, w_{m}$ be given as in Theorem 4.1 , and $w$ be a closed $q$ form, $q>1$, such that $w_{i} \wedge w=0,1 \leq i \leq m$.

Recall that, for any $\alpha:\left(I^{n}, \partial I^{n}\right) \rightarrow\left(X, x_{0}\right)$, the iterated integral $\int_{\alpha} w_{i} w_{j} \cdots w_{k} w$ is well defined and depends only on the homotopy class of $\alpha$. We give the following interpretation of this integral.

Let $\bar{X}$ be the universal covering space of $X$, and $\tilde{x}_{0}$ be a base point of $\bar{X}$ above $x_{0}$. The path integral $\int_{x_{0}} w_{i} w_{j} \cdots w_{k}$ may be taken as a function $\hat{f}$ on $\bar{X}$ with $\bar{f}\left(\tilde{x}_{0}\right)=0$. Lift $w$ to a $q$-form $\tilde{w}$ on $\bar{X}$, and $\alpha$ to $\tilde{\alpha}: I^{q} \rightarrow \tilde{X}$ such that $\tilde{\alpha}(0)$ $=\tilde{x}_{0}$. Then

$$
\int_{\alpha} w_{i} w_{j} \cdots w_{k} w=\int_{\tilde{\alpha}} \tilde{f} \tilde{w} .
$$

Let $k[[X]] Y$ be the free left $k[[X]]$-module generated by $\mathrm{Y}$. Define

$$
\Theta: \pi_{q}(X) \rightarrow k[[X]] Y
$$

by

$$
\bar{\alpha} \mapsto \int_{\alpha} w Y+\sum_{i} \int_{\alpha} w_{i} w X_{i} Y+\sum_{i, j} \int_{\alpha} w_{i} w_{j} w X_{i} X_{j} Y+\cdots
$$

Proposition. If $\bar{\alpha} \in \pi_{q}(X)$ and $\bar{\beta} \in \pi_{1}(X)$, then

$$
\Theta(\bar{\beta} \cdot \bar{\alpha})=\theta(\bar{\beta}) \Theta(\bar{\alpha}) .
$$

Proof. Let $u_{1}=w_{1_{1}}, \cdots, u_{r}=w_{i_{r}}$. We are going to show that 


$$
\begin{aligned}
\int_{\beta \cdot \alpha} u_{1} \cdots u_{r} w= & \int_{\alpha} u_{1} \cdots u_{r} w+\cdots \\
& +\int_{\beta} u_{1} \cdots u_{i} \int_{\alpha} u_{i+1} \cdots u_{r} w+\cdots+\int_{\beta} u_{1} \cdots u_{r} \int_{\alpha} w .
\end{aligned}
$$

Let $\int_{x_{0}} u_{1} \cdots u_{r}$ represent a function $\bar{g}$ on the universal covering space $\bar{X}$ with $\bar{g}\left(\tilde{x}_{0}\right)=0, \beta$ be lifted to a path from $\tilde{x}_{0}$ to $\tilde{x}_{1}$ in $\bar{X}$, and $T_{\beta}$ be the covering transformation taking $\tilde{x}_{0}$ to $\tilde{x}_{1}$. Then $\bar{g} \circ T_{\beta}$ can be represented by

$$
\int_{\beta} u_{1} \cdots u_{r}+\int_{\beta} u_{1} \cdots u_{r-1} \int_{x_{0}} u_{r}+\cdots+\int_{x_{0}} u_{1} \cdots u_{r} .
$$

Let $\tilde{w}$ be the $q$-form on $\bar{X}$ induced by $w$, and lift $\alpha$ to $\tilde{\alpha}\left(I^{q}, \partial I^{q}\right) \rightarrow\left(\tilde{X}, \tilde{x}_{0}\right)$. Then

$$
\int_{\tilde{\alpha} \cdot \tilde{\beta}} u_{1} \cdots u_{r} w=\int_{T_{\beta} \tilde{\alpha}} \tilde{g} \tilde{w}=\int_{\tilde{\alpha}}\left(\tilde{g} \circ T_{\beta}\right) \tilde{w},
$$

which is equal to the right hand side of (4.2.1).

4.3 Proposition. If $\bar{\alpha}_{1}, \bar{\alpha}_{2} \in \pi_{q}(X), q>1$, then

$$
\Theta\left(\bar{\alpha}_{1}+\bar{\alpha}_{2}\right)=\Theta\left(\bar{\alpha}_{1}\right)+\Theta\left(\bar{\alpha}_{2}\right) \text {. }
$$

Proof. What we required to show is

$$
\int_{\alpha_{1}+\bar{\alpha}_{2}} u_{1} \cdots u_{r} w=\int_{\bar{\alpha}_{1}} u_{1} \cdots u_{r} w+\int_{\alpha_{2}} u_{1} \cdots u_{r} w
$$

with $u_{1}, \cdots, u_{r}$ as given in $\S 4.2$. Write

$$
I_{1}^{q}=\left\{(t) \in I^{q}: 0 \leq t_{q} \leq \frac{1}{2}\right\}, \quad I_{2}^{q}=\left\{(t) \in I^{q}: \frac{1}{2} \leq t_{q} \leq 1\right\} .
$$

We may choose $\alpha_{1}$ and $\alpha_{2}$ such that $\alpha_{1}(t)=x_{0} \forall(t) \in I_{2}^{q}$ and $\alpha_{2}(t)=$ $x_{0} \forall(t) \in I_{1}^{q}$. Construct $\alpha:\left(I^{q}, \partial I^{q}\right) \rightarrow\left(X, x_{0}\right)$ such that $\alpha(t)=\alpha_{1}(t) \forall(t) \in I_{1}^{q}$ and $\alpha(t)=\alpha_{2}(t) \forall(t) \in I_{2}^{q}$. Then $\bar{\alpha}=\bar{\alpha}_{1}+\bar{\alpha}_{2}$. Moreover, $\left(\int_{x_{0}} u_{1} \cdots u_{r}\right)_{\alpha}(t)$ $=\left(\int_{x_{0}} u_{1} \cdots u_{r}\right)_{\alpha^{\prime}}(t) \forall(t) \in I_{i}^{q}, i=1,2$. Hence (4.3.1) holds.

For a multiplicative group $G$, denote its integral group ring by $Z G$.

4.4 Theorem. Let $X$ be a connected $C^{\infty}$ manifolds, $w_{1}, \cdots, w_{m}$ be closed 1-forms, and $w$ be a closed $q$-form on $X$ satisfying the following conditions: 
(a) $w_{i} \wedge w_{j}=0, w_{i} \wedge w=0, i, j=1, \cdots, m$,

(b) The cohomology classes $\left[w_{1}\right], \ldots,\left[w_{m}\right]$ are linearly independent.

(c) $q>1$, and there exists $\bar{\alpha} \in \pi_{q}(X)$ with $\int_{\alpha} w \neq 0$.

Then there exists a free subgroup $G$ of rank $m$ of $\pi_{1}(X)$ such that the Z-module homomorphism $\psi: Z G \rightarrow \pi_{q}(X)$ given by $\bar{\beta} \mapsto \bar{\beta} \cdot \bar{\alpha}, \forall \bar{\beta} \in G$, is injective.

Proof. According to $\S 4.1$, we may assume the existence of $\bar{\beta}_{1}, \cdots$, $\bar{\beta}_{m} \in \pi_{1}(X)$ such that $\int_{\beta j} w_{i}=\delta_{i j}$. Let $\theta$ and $\Theta$ be defined as before, and the $Z$ module homomorphism $\tilde{\theta}: Z G \rightarrow k[[X]]$ be given by $\bar{\beta} \rightarrow \theta(\bar{\beta}), \forall \bar{\beta} \in G$.

We claim that $\theta(\bar{\beta})$ is a grouplike element of $k[[X]]$ as defined in $\S 3.5$. In fact,

$$
\begin{aligned}
\Delta \theta(\bar{\beta}) & =1+\cdots+\sum \int_{\beta} w_{i_{1}} \cdots w_{i_{r}}\left(X_{i_{1}}^{\prime}+\cdots X_{i_{r}}^{\prime \prime}\right) \cdots\left(X_{i_{r}}^{\prime}+X_{i_{r}}^{\prime \prime}\right)+\cdots \\
& =1+\cdots+\sum_{\sigma} \int_{\beta} w_{i_{\sigma}(i)} \cdots w_{i_{r(r+s)}} X_{i_{1}}^{\prime} \cdots X_{i_{r}}^{\prime} X_{i_{r+1}}^{\prime \prime} \cdots X_{i_{r+1}}^{\prime \prime}+\cdots
\end{aligned}
$$

where the summation is taken over all $(r, s)$-shuffles $\sigma$. It follows from (3.4.2) that

$$
\Delta \theta(\bar{\beta})=\left(\rho^{\prime} \theta(\bar{\beta})\right)\left(\rho^{\prime \prime} \theta(\bar{\beta})\right) .
$$

According to $\S 4.1, \theta$ restricted to $G$ is injective. Thus Proposition 3.5 implies that $\tilde{\theta}$ is injective.

Observe that $\Theta(\bar{\alpha})=\eta Y$ where

$$
\eta=\int_{\alpha} w+\sum_{i} \int_{\alpha} w_{i} w X_{i}+\cdots
$$

is a unit in the ring $k[[X]]$ because $\int_{\alpha} w \neq 0$. Therefore we may regard $k[[X]] Y$ as a free $k[[X]]$-module with $\Theta(\bar{\alpha})$ as a generator. Since $\theta(\bar{\beta}), \bar{\beta} \in G$, are linearly independent, so are $\Theta(\bar{\beta} \cdot \bar{\alpha})=\theta(\bar{\beta}) \Theta(\bar{\alpha}), \bar{\beta} \in G$. Hence $\bar{\beta} \cdot \bar{\alpha}, \bar{\beta} \in G$, are linearly independent over $Z$ in $\pi_{q}(X)$.

Corollary. Let $X$ be a compact Kähler manifold. If $b^{1,0}>b^{2,0}+b^{q+1,0}+1$, $q>1$, and the integral of some closed holomorphic $q$-form, $q>1$, over $a$ spherical q-chain is not zero, then $\pi_{q}(X)$ contains a free abelian subgroup of infinite rank.

Proof. Set $s=b^{q+1,0}$ and $l=b^{1,0}-s$. There must exist $l$ linearly independent close holomorphic 1 -forms $w_{1}, \cdots, w_{l}$ such that $w_{i} \wedge w=0, i=1, \cdots, l$. Since $l>b^{2,0}+1$, we may use the same argument as in the proof of Corollary 4.1 
to obtain $w_{1} \wedge w_{2}=0$. Hence the theorem can be applied.

The next assertion follows from the proof of the theorem.

Corollary. If a compact Kähler manifold $X$ of complex dimension $n>1$ has a nonconstant holomorphic map into a Riemann surface of genus $>1$, and $\pi_{n}(X)$ is finitely generated, then the integral of every holomorphic $n$-form of $X$ must vanish over any spherical $n$-chain.

4.5. If $H^{2}(X)=0$ for a connected $C^{\infty}$ manifold, it can be easily verified that, for any given closed 1 -forms $w_{1}, \cdots, w_{r}$, there exists an iterated integral of the type $\left\langle w_{1}, \cdots, w_{r}\right\rangle$ as defined in $\S 2.2$. Using this fact, it can be shown that $\pi_{1}(X)$ has a free subgroup of rank equal to the first Betti number of $X$.

4.6. So far, we have given no applications of iterated integrals purely involving forms of higher degrees. J. H. C. Whitehead's integral (see also [9]) for Hopf fibering of spheres can be taken as a twice iterated integral as explained below.

Let $f: S^{2 n-1} \rightarrow S^{n}, n=2,4,8$, be the Hopf fibering, and $w$ be a closed $n$-form on $S^{n}$ such that $\int_{S^{n}} w=1$. Take a base point $p_{0} \in S^{2 n-1}$. Let $\alpha$ be the composite map

$$
\left(I^{2 n-1}, \partial I^{2 n-1}\right) \stackrel{g}{\longrightarrow}\left(S^{2 n-1}, p_{0}\right) \stackrel{f}{\longrightarrow}\left(S^{n}, f p_{0}\right)
$$

where $g$ is a $C^{\infty}$-map which induces an isomorphism

$$
\pi_{2 n-1}\left(I^{2 n-1}, \partial I^{2 n-1}\right) \approx \pi_{2 n-1}\left(S^{2 n-1}\right) .
$$

Let $v$ be a $(n-1)$-form in $S^{2 n-1}$ such that $d v=f^{*} w$. Then

$$
\int_{\alpha} w w=\int_{I^{2 n-1}} g^{*} v \wedge \alpha^{*} w=\int_{S^{2 n-1}} v \wedge f^{*} w,
$$

which yields the Hopf invariant.

\section{Bibliography}

[1] K. T. Chen, Interated integrals and exponential homomorphisms, Proc. London Math. Soc. (3) 4 (1954) 502-512.

[2] - Algebras of interated path integrals and fundamental groups, Trans. Amer. Math. Soc. 156 (1971) 359-379.

[ 3 ] S. S. Chern, Complex manifolds, Mimeographed notes, University of Chicago, 1955-1956.

[ 4 ] D. Kraines, Massey higher products, Trans. Amer. Math. Soc. 124 (1966) 431449.

[ 5 ] W. Magnus, A. Karrass \& D. Solitar, Combinatorial group theory, Wiley, New York, 1966.

[ 6] W. S. Massey, Some higher order cohomology operations, Sympos. Internac. Topología Algebraica, Mexico, 1958, 145-154. 
[7] J. Milnor, Isotopy of links, Algebraic Geometry and Topology, A symposium in Honor of S. Lefschetz, Princeton University Press, Princeton, 1957, 280-306.

[ 8 ] J. Stallings, Homology and central series of groups, J. Algebra 2 (1965) 170-181.

[9] N. E. Steenrod, Cohomology invariants of mappings, Ann. of Math. 50 (1949) 954-988.

[10] M. E. Sweedler, Hopf algebras, Benjamin, New York, 1969.

[11] A. Weil, Variétés kählériennes, Hermann, Paris, 1958.

[12] J. H. C. Whitehead, An expression of Hopf's invariant as an integral, Proc. Nat. Acad. Sci. U.S.A. 33 (1947) 117-123.

UNIVERSITY OF ILLINOIS 